\title{
LOWER BOUNDS FOR QUASIANALYTIC FUNCTIONS, I. HOW TO CONTROL SMOOTH FUNCTIONS
}

\author{
F. NAZAROV, M. SODIN* and A. VOLBERG \\ In memory of Thøger Bang (1917-1997)
}

\begin{abstract}
Let $\mathscr{F}$ be a class of functions with the uniqueness property: if $f \in \mathscr{F}$ vanishes on a set $E$ of positive measure, then $f$ is the zero function. In many instances, we would like to have a quantitative version of this property, e.g. the estimate from below for the norm of the restriction operator $\left.f \mapsto f\right|_{E}$ or, equivalently, a lower bound for $|f|$ outside a small exceptional set. Such estimates are well-known and useful for polynomials, complex- and real-analytic functions, exponential polynomials. In this work we prove similar results for the Denjoy-Carleman and the Bernstein classes of quasianalytic functions.

In the first part, we consider quasianalytically smooth functions. This part relies upon Bang's approach and includes the proofs of relevant results of Bang. In the second part, which is to be published separately, we deal with classes of functions characterized by exponentially fast approximation by polynomials whose degrees belong to a given very lacunar sequence.

The proofs are based on the elementary calculus technique.
\end{abstract}

\section{Motivation and the results}

Let $P$ be a polynomial. Its degree $d$ governs the behaviour of $P$ on any interval $I \subset \mathrm{R}$, for instance, $P$ has at most $d$ zeroes on $I$ and for any measurable subset $E \subset I$

$$
\|P\|_{I} \leq\left(\frac{4|I|}{|E|}\right)^{d}\|P\|_{E} .
$$

Here and everywhere below, $\|f\|_{K}=\sup _{K}|f|$ denotes the supremum norm on $K$, and $|E|$ denotes the length of a set $E \subset \mathrm{R}$.

The first fact hardly needs any comments. The second one is a rough version of the classical Remez inequality [19] (cf. [8] and [4]). Different proofs of (1.1) are known. The simplest one uses the Lagrange interpolation formula for $P$

\footnotetext{
* The author was supported by the Israel Science Foundation of the Israel Academy of Sciences and Humanities under Grant No. 37/00-1.

Received March 26, 2003.
} 
with $d+1$ nodes on $E$ spaced by at least $|E| / d$, though this gives us (1.1) with a worse constant $2 e$ instead of 4 on the right hand side [16]. The Remez inequality has a plenty of applications and extensions, some of them can be found in [8], [4], [16], [17]; by no means is this list complete. The inequality is sufficiently sharp to capture that $P$ cannot have zeroes of multiplicity larger than $d$.

Turning to analytic functions, we encounter another quantity which controls their behaviour. Let $G \subset C^{1}$ be a bounded domain, $K \subset G$ be a compact subset, and let $f$ be a bounded analytic function in $G$. Then the logarithm of the ratio

$$
\mathfrak{B}_{f}(K, G)=\log \frac{\|f\|_{G}}{\|f\|_{K}}
$$

is called the Bernstein degree of $f$ on $(K, G)$. If $P$ is polynomial of degree $d$, and $G_{R} \subset C^{1}$ is the ellipse with the foci at $-1,+1$ and the semiaxes $R$, then by the classical Bernstein inequality

$$
\mathfrak{B}_{P}\left([-1,1], G_{R}\right) \leq d \log R .
$$

The Bernstein degree controls the number of zeroes of $f$ on $K$ as well as the local oscillations of $f$. By the Jensen formula the number of zeroes of $f$ on $K$ counting with multiplicities does not exceed $\gamma(K, G) \mathfrak{B}_{f}(K, G)$ where $\gamma(K, G)$ depends only on the geometry of the couple $(K, G)$. The Cartan lemma yields local estimates on $K$ similar to (1.1) with the exponent $\gamma(K, G) \mathfrak{B}_{f}(K, G)$. The interest to this classical theme was recently revived (cf. [5], [9], [10], [21], [24] and the references therein).

In this work we shall exhibit a new index which controls in a similar fashion the behaviour of quasianalytically smooth functions.

Given closed interval $J \subset \mathrm{R}$ and given a sequence of positive numbers $\left\{M_{j}\right\}$, introduce the class $\mathscr{C}_{\left\{M_{j}\right\}}(J)$ of $C^{\infty}(J)$-functions such that

$$
\left\|f^{(j)}\right\|_{J} \leq M_{j}, \quad j \in \mathbf{Z}_{+} .
$$

We assume that the sequence $\left\{M_{j}\right\}$ is logarithmically convex, that is

$$
M_{j}^{2} \leq M_{j-1} M_{j+1}, \quad j \in \mathrm{N} .
$$

A convenient way to generate logarithmically convex sequences is to fix a non-decreasing function $A:[1, \infty) \rightarrow(0, \infty)$ and set

$$
M_{j}=M_{j-1} A(j), \quad j \geq 1 .
$$

Rescaling the argument of $f$ and multiplying $f$ by a constant, we can always assume that $f$ is defined on the interval $J=[0,1]$ and that $M_{0}=1$. Usually, 
we shall keep this normalization and denote the normalized Denjoy-Carleman classes by $\mathscr{C}_{A}([0,1])$.

According to the classical Denjoy-Carleman theorem [6], the divergence of the series

$$
\sum_{j=1}^{\infty} \frac{M_{j-1}}{M_{j}}=+\infty
$$

(or equivalently of the integral $\int_{1}^{\infty} \frac{d t}{A(t)}=+\infty$ ) is a necessary and sufficient condition for quasianalyticity of the class $\mathscr{C}_{\left\{M_{j}\right\}}(J)$ [6] (that is, $\mathscr{C}_{\left\{M_{j}\right\}}(J)$ contains no non-trivial function which vanishes at a point with all derivatives). In the paper [2] published 50 years ago, Bang gave an intrinsic and elementary real variable proof of the uniqueness part of the Denjoy-Carleman theorem. Strangely enough, this concise paper left no trace in the vast literature devoted to quasianalytic functions, unlike Bang's thesis [1] which appeared to be more influential (cf. [14, Chapter IV], [7], [12, Section 1.3]). For this reason, we took a liberty to reproduce (with minor variations) some results from [2] with their proofs.

Definition. The Bang degree $\mathfrak{n}_{f}$ of the function $f \in \mathscr{C}_{A}([0,1])$ is the largest integer $N$ such that

$$
\sum_{\log \|f\|_{[0,1]}^{-1}<j \leq N} \frac{M_{j-1}}{M_{j}}<e
$$

If the set of positive integers $N$ satisfying (1.6) is unbounded, then we set formally $\mathfrak{n}_{f}=+\infty$.

After a minute reflection, one can see a certain similarity between the Bernstein and the Bang degrees. The latter depends on the decay of the sequence $\left\{M_{j-1} / M_{j}\right\}$ (that is, on the a priori smoothness of $f$ ), and on the lower bound for $\|f\|_{[0,1]}$ (the closer is $\|f\|_{[0,1]}$ to its a priori upper bound $M_{0}=1$, the smaller is the degree $\left.\mathfrak{n}_{f}\right)$. If the series (1.5) diverges, that is the class $\mathscr{C}_{A}([0,1])$ is quasianalytic, then the degree $\mathfrak{n}_{f}$ is always finite. In the non-quasianalytic case, the degree can be infinite. In fact, we can allow the function $f$ to have only finite smoothness: if $f \in C^{m}([0,1])$, then we simply put $A(j)=+\infty$ starting with $j=m+1$.

TheOREM A (Bang [2]). The total number of zeroes (counting with multiplicities) of any function $f \in \mathscr{C}_{A}([0,1])$ does not exceed its Bang degree $\mathfrak{n}_{f}$. 
The first result of that type was conjectured by Borel and proved by Carleman in [6, p.24-27]. Carleman based the proof on the Fourier transform and harmonic estimation. The theorem says that if $f \in C^{n}([0,1])$ and satisfies

$$
f^{(j)}(0)=0, \quad 0 \leq j \leq n-1, \quad \text { and } \quad f(1)=1,
$$

then

$$
\sum_{j=1}^{n} \frac{1}{M_{j}^{1 / j}}<8\left(\frac{1}{2}+\pi e+2 \sqrt{\pi e}\right)
$$

where $M_{j}=\left\|f^{(j)}\right\|_{[0,1]}$.

This estimate cannot be deduced directly from Theorem A since the sequence $\left\{M_{j}\right\}$ we deal with is assumed to be logarithmically convex whereas Borel and Carleman did not impose any condition on that sequence. Nevertheless, as we shall see in subsection 5.4, there is a more general version of Bang's result which contains the result of Carleman.

One can probably extract from Hirschman's paper [11] a result similar to Theorem A (even with $\frac{2}{\pi}$ instead of $e$ on the right hand side of (1.6)), however with some additional regularity of the sequence $\left\{M_{j}\right\}$. Hirschman used the Carleman technique combined with the Cartan-Gorny estimates of derivatives of smooth functions.

In the second theorem it will be convenient to assume that the function $A$ which defines according to (1.4) the sequence $\left\{M_{j}\right\}$ is a $C^{1}$-function (if $A$ is a piecewise linear function, then one can use the left derivative). We set

$$
\gamma(n):=\sup _{1 \leq s \leq n} \frac{s A^{\prime}(s)}{A(s)}
$$

and

$$
\Gamma(n)=4 e^{4+\gamma(n)} .
$$

Theorem B. Suppose $f \in \mathscr{C}_{A}([0,1])$. Then for any interval $I \subset[0,1]$ and any measurable subset $E \subset I$

$$
\|f\|_{I} \leq\left(\frac{\Gamma\left(2 \mathfrak{n}_{f}\right)|I|}{|E|}\right)^{2 \mathfrak{n}_{f}}\|f\|_{E}
$$

We say that the class $\mathscr{C}_{A}([0,1])$ is regular if the supremum

$$
\gamma:=\sup _{s \geq 1} \frac{s A^{\prime}(s)}{A(s)}
$$


is finite. For example, the real analytic class $(A(s)=s)$ and the logarithmic classes $\left(A(s)=s \log ^{\alpha}(s+e)\right)$ are regular. For regular classes, estimate (1.8) holds with the factor $\Gamma=4 e^{4+\gamma}$ on the right hand side.

Theorems A and B show that Bang's degree is an important characteristics of smooth functions. However, we do not know much about its basic properties. For example, if $f$ is a polynomial, how to bound from above the Bang degree $\mathfrak{n}_{f}$ by the usual degree? If $f$ is real analytic, the same question can be asked about the upper bound of the Bang degree by the Bernstein degree. Recently, N. Roytvarf [20] and D. Novikov and S. Yakovenko [18] obtained useful estimates for the Bernstein degree of linear combinations, products, (analytic) quotients and derivatives of given functions. It seems to be interesting to get results in that spirit for the Bang degree. At last, it looks probable, that Bang's degree has a certain invariance under real analytic diffeomorphisms of the interval $[0,1]$.

ACKNOWLEDGment. The authors thank Alexander Borichev and Iossif Ostrovskii for numerous useful remarks.

\section{Bang's fundamental inequality}

Given a function $f \in \mathscr{C}_{A}([0,1])$ and a point $x \in[0,1]$, we define the norm of $f$ at $x$ as

$$
B_{f}(x):=\max _{j \geq 0} \frac{\left|f^{(j)}(x)\right|}{e^{j} M_{j}} .
$$

A small norm means that a large section of the sequence $\left\{\frac{\left|f^{(j)}(x)\right|}{M_{j}}\right\}_{j \geq 0}$ consists of small numbers. For example, $B_{f}(x) \leq e^{-q}$ for some $q \in \mathbf{Z}_{+}$if and only if $\left|f^{(j)}(x)\right| \leq e^{j-q} M_{j}$ for $0 \leq j \leq q$.

Lemma 2.1 (Bang). For any $q \in \mathrm{N}$ and any $x, x+h \in[0,1]$,

$$
B_{f}(x+h)<\max \left\{B_{f}(x), e^{-q}\right\} e^{e|h| A(q)} .
$$

Proof of the lemma. We fix $j$ in the range $0 \leq j \leq q-1$ and find $\xi$ between $x$ and $x+h$ such that

$$
f^{(j)}(x+h)=\sum_{l=0}^{q-j-1} \frac{f^{(j+l)}(x) h^{l}}{l !}+\frac{f^{(q)}(\xi) h^{q-j}}{(q-j) !} .
$$


Then

$$
\begin{aligned}
& \frac{\left|f^{(j)}(x+h)\right|}{e^{j} M_{j}} \\
& \quad \leq \sum_{l=0}^{q-j-1} \frac{\left|f^{(j+l)}(x)\right||h|^{l}}{e^{j} M_{j} l !}+\frac{\left|f^{(q)}(\xi)\right||h|^{q-j}}{e^{j} M_{j}(q-j) !} \\
& \quad=\sum_{l=0}^{q-j-1} \frac{\left|f^{(j+l)}(x)\right|}{e^{j+l} M_{j+l}} \frac{M_{j+l}}{M_{j}} \frac{e^{l}|h|^{l}}{l !}+e^{-q} \frac{\left|f^{(q)}(\xi)\right|}{M_{q}} \frac{M_{q}}{M_{j}} \frac{|h|^{q-j} e^{q-j}}{(q-j) !} \\
& \quad(1.3) \\
& \quad \leq B_{f}(x) \sum_{l=0}^{q-j-1}\left(\frac{M_{q}}{M_{q-1}}\right)^{l} \frac{e^{l}|h|^{l}}{l !}+e^{-q}\left(\frac{M_{q}}{M_{q-1}}\right)^{q-j} \frac{|h|^{q-j} e^{q-j}}{(q-j) !} \\
& \quad<\max \left\{B_{f}(x), e^{-q}\right\} \exp \left(e|h| \frac{M_{q}}{M_{q-1}}\right) \cdot
\end{aligned}
$$

If $j \geq q$, the same estimate holds for a trivial reason:

$$
\frac{\left|f^{(j)}(x+h)\right|}{e^{j} M_{j}} \leq e^{-q}<\max \left(B_{f}(x), e^{-q}\right) e^{e|h| A(q)},
$$

completing the argument.

Corollary 2.3. Suppose $f \in \mathscr{C}_{A}([0,1])$. If

$$
\max _{[0,1]} B_{f} \geq e^{-L}
$$

and

$$
\min _{[0,1]} B_{f} \leq e^{-N}
$$

then

$$
\sum_{L+1 \leq j \leq N} \frac{M_{j-1}}{M_{j}}<e
$$

Proof of the corollary. Let $B_{f}\left(x_{N}\right)=e^{-N}$ and $B_{f}\left(x_{L}\right)=e^{-L}$. By (2.2), the function $x \mapsto B_{f}(x)$ is continuous on $[0,1]$. Therefore, we can choose a monotonic sequence $\left\{x_{j}\right\}_{L \leq j \leq N} \subset J$ such that $B_{f}\left(x_{j}\right)=e^{-j}$ for $L \leq j \leq N$. By Lemma 2.1,

$$
\left|x_{j}-x_{j-1}\right|>\frac{1}{e} \frac{M_{j-1}}{M_{j}},
$$


so that

$$
1 \geq \sum_{L+1 \leq j \leq N}\left|x_{j}-x_{j-1}\right|>\frac{1}{e} \sum_{L+1 \leq j \leq N} \frac{M_{j-1}}{M_{j}}
$$

proving the corollary.

If the function $f \in \mathscr{C}_{A}([0,1])$ has a zero of order at least $N$ at some point $x_{0} \in[0,1]$ (that is, $f\left(x_{0}\right)=f^{\prime}\left(x_{0}\right)=\ldots=f^{(N-1)}\left(x_{0}\right)=0$ ), then $B_{f}\left(x_{0}\right) \leq e^{-N}$. On the other hand,

$$
\max _{[0,1]} B_{f} \geq\|f\|_{[0,1]} .
$$

Then the corollary says that the order $N$ of any zero of $f$ is bounded from above by the Bang degree $\mathfrak{n}_{f}$. This is a version of a theorem of Borel and Carleman mentioned above. In particular, the uniqueness part of the DenjoyCarleman theorem follows at once: non-trivial functions $f$ from the quasianalytic Denjoy-Carleman class $\mathscr{C}_{A}([0,1])$ cannot have a zero of infinite order.

In the non-quasianalytic case, when

$$
\sum_{j=1}^{\infty} \frac{M_{j-1}}{M_{j}}<\infty
$$

rescaling estimate (2.6), we get an upper bound for the function $f$ near its zeroes of infinite order.

Corollary 2.7. Suppose $f \in \mathscr{C}_{A}([0,1])$. Let

$$
f^{(j)}(0)=0, \quad j \in \mathbf{Z}_{+} .
$$

Then

$$
\sum_{j \geq \log M_{f}^{-1}(c)+1} \frac{M_{j-1}}{M_{j}}<e c
$$

where

$$
\mathfrak{M}_{f}(c)=\max _{x \in[0, c]}|f(x)|
$$

Under additional regularity assumptions on the function $A$, Matsaev and Sodin recently found in [15] the sharp asymptotics for

$$
\log \sup _{f} \mathfrak{M}_{f}(c), \quad c \rightarrow 0,
$$

where the supremum is taken over all functions $f \in \mathscr{C}_{\left\{M_{j}\right\}}(\mathrm{R})$ with $M_{0}=1$, having the zero of infinite order at the origin. 


\section{Proof of Theorem A}

Now, we consider a sequence of 'norms' obtained from the remainders:

$$
b_{f, n}(x)=\max _{j \geq n} \frac{\left|f^{(j)}(x)\right|}{e^{j} M_{j}}=e^{-n} B_{f^{(n)}}(x),
$$

here $f^{(n)}$ is considered in the class $\mathscr{C}_{\left\{M_{n}, M_{n+1}, \ldots\right\}}([0,1])$. List some properties of this sequence:

(i) $b_{f, n}(x) \leq e^{-n}$;

(ii) $B_{f}(x)=b_{f, 0}(x) \geq b_{f, 1}(x) \geq \cdots \geq b_{f, n}(x) \geq \cdots$;

(iii) if $f^{(n)}\left(x^{*}\right)=0$, then $b_{f, n}\left(x^{*}\right)=b_{f, n+1}\left(x^{*}\right)$;

(iv) the function $b_{f, n}$ satisfies the estimate

$$
b_{f, n}(x+h) \leq \max \left\{b_{f, n}(x), e^{-q-n}\right\} e^{e|h| A(q)},
$$

for every $q \in \mathrm{N}$ and every $x, x+h \in[0,1]$.

From the last property we conclude that

(v) the function $x \mapsto b_{f, n}(x)$ is continuous on $[0,1]$;

(vi) $b_{f, n}(x+h)<e^{-j+1}$ provided $b_{f, n}(x) \leq e^{-j}$ and $e|h| A(j) \leq 1$.

The latter is interesting only for $j>n$.

After these preliminaries we start the proof. Let $x_{*} \in[0,1]$ be the maximum point of $B_{f}$ :

$$
\max _{[0,1]} B_{f}=B_{f}\left(x_{*}\right) .
$$

First, we consider a special case, when $x_{*}$ is one of the end-points of $[0,1]$. Without loss of generalities, suppose that $x_{*}=0$. Let

$$
0<\xi_{1} \leq \cdots \leq \xi_{N}
$$

be the zeroes of $f$ on $[0,1]$ counted with their multiplicities. Applying Rolle's theorem, we find another $N$-point set $\left\{x_{j}\right\}_{0 \leq j \leq N-1}$, such that

$$
f^{(j)}\left(x_{j}\right)=0, \quad \text { for } \quad 0 \leq j \leq N-1,
$$

$x_{0}=\xi_{1}$, and $x_{j} \leq \xi_{j+1}$. Then we can paste together the functions $b_{f, j}$ with different $j$ and define the new function

$$
b_{f}(x)= \begin{cases}b_{f, 0}(x), & 0 \leq x<x_{0} \\ b_{f, 1}(x), & x_{0} \leq x<x_{1} \\ \ldots \ldots \ldots \ldots \ldots \ldots \ldots \ldots & \ldots \ldots \ldots \ldots \\ b_{f, N-1}(x), & x_{N-2} \leq x<x_{N-1} \\ b_{f, N}(x), & x \geq x_{N-1} .\end{cases}
$$


This is a continuous function with the following properties:

$$
\begin{gathered}
b_{f}(0)=B_{f}(0) \geq\|f\|_{[0,1]}, \\
b_{f}(x) \leq e^{-N}, \quad \text { for } \quad x \geq x_{N-1},
\end{gathered}
$$

and $b_{f}(x+h)<e^{-j+1}$ provided that $b_{f}(x) \leq e^{-j}$ and $e h A(j) \leq 1, h>0$.

Computing as above, in the proof of Corollary 2.3, the number of level crossings of the function $b_{f}$ we obtain

$$
1>\frac{1}{e} \sum_{j=K_{f}+1}^{N} \frac{M_{j-1}}{M_{j}}
$$

where $K_{f}=\left\lfloor\left|\log \|f\|_{[0,1]}\right|\right\rfloor$. Thereby $N \leq \mathfrak{n}_{f}$, completing the proof in the special case.

Now, consider the general case. If $x_{*}$ is not the end-point of [0,1], then $x_{*}$ splits $[0,1]$ into two subintervals $J_{1}$ and $J_{2}$ on which $f$ has $N_{1}$ and $N_{2}$ zeroes respectively, $N_{1}+N_{2}=N$. By the special case proven above (rescaling the argument of $f$ ) we have

$$
\left|J_{l}\right|>\frac{1}{e} \sum_{j=K_{f}+1}^{N_{l}} \frac{M_{j-1}}{M_{j}}, \quad l=1,2 .
$$

At last, making use of the logarithmic convexity of the sequence $\left\{M_{j}\right\}$ we obtain

$$
\begin{aligned}
1=\left|J_{1}\right|+\left|J_{2}\right|>\frac{1}{e}\left\{\sum_{j=K_{f}+1}^{N_{1}}+\sum_{j=K_{f}+1}^{N_{2}}\right\} \frac{M_{j-1}}{M_{j}} \\
\quad \geq \frac{1}{e}\left\{\sum_{j=K_{f}+1}^{N_{1}}+\sum_{j=N_{1}+1}^{N_{1}+N_{2}}\right\} \frac{M_{j-1}}{M_{j}}=\frac{1}{e} \sum_{j=K_{f}+1}^{N} \frac{M_{j-1}}{M_{j}}
\end{aligned}
$$

whence $N \leq \mathfrak{n}_{f}$. This completes the proof of Theorem A.

\section{Proof of Theorem B}

We put $m_{0}=M_{0}=1$ and

$$
m_{j}=\frac{M_{j}}{j !}, \quad j \in \mathrm{N} .
$$


We shall prove Theorem B in three steps. First, we prove a preliminary version of estimate (1.8) with a remainder term:

$$
\|f\|_{I} \leq\left(\frac{2 e|I|}{|E|}\right)^{n}\|f\|_{E}+m_{n+1}|I|^{n+1}
$$

for each $n \in \mathrm{N}$. Then we shall show that if the interval $I$ is sufficiently short, then the remainder $m_{2 \mathfrak{n}_{f}}|I|^{2 \mathfrak{n}_{f}}$ is smaller than the norm $\|f\|_{I}$ we are estimating. Combined with (4.1) this gives us estimate (1.8) for short intervals $I$. At the last step, we shall extend estimate (1.8) to arbitrary intervals $I \subset[0,1]$.

Proof of estimate (4.1). We choose well-spaced points $\left\{x_{j}\right\}_{j=1}^{n+1} \subset E$,

$$
x_{1}<x_{2}<\cdots<x_{n+1}, \quad \min _{j}\left(x_{j+1}-x_{j}\right) \geq \frac{|E|}{n},
$$

and set

$$
Q(x)=\prod_{j=1}^{n+1}\left(x-x_{j}\right)
$$

Then for $x \in I$

$$
f(x)=\sum_{j=1}^{n+1} \frac{f\left(x_{j}\right) Q(x)}{Q^{\prime}\left(x_{j}\right)\left(x-x_{j}\right)}+\frac{f^{(n+1)}(\xi) Q(x)}{(n+1) !}, \quad \xi=\xi_{x} \in I .
$$

This is a well-known version of the Lagrange interpolation formula. The proof goes as follows: fix $x \in I$ and consider the function

$$
G(t)=Q(t) R(x)-Q(x) R(t)
$$

where $R(t)$ is the remainder; i.e. the difference between $f$ and the Lagrange interpolation polynomial of degree $n$ with the nodes at $\left\{x_{j}\right\}$. The function $G(t)$ has at least $n+2$ zeroes on $I$ : it vanishes at $n+1$ points: $t=x_{j}$ and also at $t=x$. Therefore, the derivative $G^{(n+1)}(t)$ vanishes at least once on $I$ :

$$
\begin{aligned}
0=G^{(n+1)}(\xi) & =Q^{(n+1)}(\xi) R(x)-Q(x) R^{(n+1)}(\xi) \\
& =(n+1) ! R(x)-Q(x) f^{(n+1)}(\xi),
\end{aligned}
$$

proving (4.2).

Then using the estimates $\|Q\|_{I} \leq|I|^{n+1}$ and

$$
\left\|\frac{Q(x)}{x-x_{j}}\right\|_{I} \leq|I|^{n}
$$


we get

$$
\|f\|_{I} \leq\left(\sum_{j=1}^{n+1} \frac{1}{\left|Q^{\prime}\left(x_{j}\right)\right|}\right)|I|^{n}\|f\|_{E}+m_{n+1}|I|^{n+1} .
$$

Further,

$$
\begin{aligned}
\left|Q^{\prime}\left(x_{j}\right)\right| & =\left(x_{j}-x_{j-1}\right) \ldots\left(x_{j}-x_{1}\right)\left(x_{j+1}-x_{j}\right) \ldots\left(x_{n+1}-x_{j}\right) \\
& \geq \frac{(j-1) !(n+1-j) !}{n^{n}}|E|^{n}>\frac{(j-1) !(n+1-j) !}{n !}\left(\frac{|E|}{e}\right)^{n},
\end{aligned}
$$

so that

$$
\sum_{j=1}^{n+1} \frac{1}{\left|Q^{\prime}\left(x_{j}\right)\right|}<\left(\frac{2 e}{|E|}\right)^{n}
$$

and (4.1) follows.

We shall use estimate (4.1) with $n=2 \mathfrak{n}_{f}-1$.

\section{Lemma 4.3. Suppose}

$$
m_{2 \mathfrak{n}_{f}}|I|^{2 \mathfrak{n}_{f}} \leq e^{-2 \mathfrak{n}_{f}\left(3+\gamma\left(2 \mathfrak{n}_{f}\right)\right)}
$$

Then

$$
m_{2 \mathfrak{n}_{f}}|I|^{2 \mathfrak{n}_{f}}<\frac{1}{2}\|f\|_{I}
$$

Now, combining estimates (4.1) and (4.5) we get

COROLlary 4.6. Suppose the interval I is short; i.e. estimate (4.4) is valid. Then

$$
\|f\|_{I} \leq 2\left(\frac{2 e|I|}{|E|}\right)^{2 \mathfrak{n}_{f}}\|f\|_{E}
$$

Proof of Lemma 4.3. follows from two claims:

Claim 4.8. Estimate (4.4) yields

$$
m_{2 \mathfrak{n}_{f}}|I|^{2 \mathfrak{n}_{f}} \leq e^{-4 \mathfrak{n}_{f}} m_{k}|I|^{k}
$$

for each $k, 0 \leq k \leq \mathfrak{n}_{f}$.

Claim 4.10. There exists $k, 0 \leq k \leq \mathfrak{n}_{f}$, such that

$$
m_{k}\left(\frac{|I|}{2}\right)^{k} \leq e^{\left(2+\frac{1}{e}\right) \mathfrak{n}_{f}}\left[\|f\|_{I}+m_{2 \mathfrak{n}_{f}}\left(\frac{|I|}{2}\right)^{2 \mathfrak{n}_{f}}\right] .
$$


First, we finish off the proof of Lemma 4.3 and then will prove the claims. Putting the claims together, we get

$$
\begin{aligned}
m_{2 \mathfrak{n}_{f}}|I|^{2 \mathfrak{n}_{f}} & \leq e^{-4 \mathfrak{n}_{f}} \cdot 2^{\mathfrak{n}_{f}} \cdot e^{\left(2+\frac{1}{e}\right) \mathfrak{n}_{f}}\left[\|f\|_{I}+m_{2 \mathfrak{n}_{f}}\left(\frac{|I|}{2}\right)^{2 \mathfrak{n}_{f}}\right] \\
& \leq e^{-0.9 \mathfrak{n}_{f}}\left[\|f\|_{I}+\frac{1}{4} m_{2 \mathfrak{n}_{f}}|I|^{2 \mathfrak{n}_{f}}\right]
\end{aligned}
$$

whence

$$
2 m_{2 \mathfrak{n}_{f}}|I|^{2 \mathfrak{n}_{f}}<\left(e^{0.9}-\frac{1}{4}\right) m_{2 \mathfrak{n}_{f}}|I|^{2 \mathfrak{n}_{f}} \leq\left(e^{0.9 \mathfrak{n}_{f}}-\frac{1}{4}\right) m_{2 \mathfrak{n}_{f}}|I|^{2 \mathfrak{n}_{f}} \leq\|f\|_{I},
$$

proving the lemma.

Proof of Claim 4.8. is straightforward. We have

$$
\frac{m_{2 \mathfrak{n}_{f}}}{m_{k}}|I|^{2 \mathfrak{n}_{f}-k} \stackrel{(4.4)}{\leq} e^{-\left(2 \mathfrak{n}_{f}-k\right)\left(3+\gamma\left(2 \mathfrak{n}_{f}\right)\right)} \frac{m_{2 \mathfrak{n}_{f}}^{k / 2 \mathfrak{n}_{f}}}{m_{k}} .
$$

Therefore, we need to estimate the expression

$$
\left(\frac{m_{2 \mathfrak{n}_{f}}^{k /\left(2 \mathfrak{n}_{f}\right)}}{m_{k}}\right)^{\frac{1}{2 \mathfrak{n}_{f}-k}}=\frac{m_{2 \mathfrak{n}_{f}}^{\frac{1}{2 n_{f}-k}-\frac{1}{2 \mathfrak{n}_{f}}}}{m_{k}^{\frac{1}{2 \mathfrak{n}_{f}-k}}}
$$

Taking the logarithm and setting $a(s)=\frac{A(s)}{s}$, that is $m(k)=a(1) a(2) \ldots a(k)$, we obtain

$$
\begin{gathered}
\frac{1}{2 \mathfrak{n}_{f}-k} \sum_{j=k+1}^{2 \mathfrak{n}_{f}} \log a(j)-\frac{1}{2 \mathfrak{n}_{f}} \sum_{j=1}^{2 \mathfrak{n}_{f}} \log a(j) \\
=\frac{1}{2 \mathfrak{n}_{f}-k} \sum_{j=k+1}^{2 \mathfrak{n}_{f}}[\log a(j)-\log a(1)]-\frac{1}{2 \mathfrak{n}_{f}} \sum_{j=1}^{2 \mathfrak{n}_{f}}[\log a(j)-\log a(1)] \\
=\int_{1}^{2 \mathfrak{n}_{f}} \frac{a^{\prime}}{a}(s)\left\{\frac{1}{2 \mathfrak{n}_{f}-k} \sum_{j=k+1}^{2 \mathfrak{n}_{f}} \chi_{[1, j]}-\frac{1}{2 \mathfrak{n}_{f}} \sum_{j=1}^{2 \mathfrak{n}_{f}} \chi_{[1, j]}\right\}(s) d s
\end{gathered}
$$

where $\chi_{[a, b]}(s)$ is the indicator function of the interval $[a, b]$. Since

$$
0 \leq\{\ldots\}(s)<\frac{s}{2 \mathfrak{n}_{f}},
$$


then we get

$$
\text { the RHS of }(4.12)<\frac{1}{2 \mathfrak{n}_{f}} \int_{1}^{2 \mathfrak{n}_{f}} \frac{s a^{\prime}(s)}{a(s)} d s \leq \sup _{s \geq 1} \frac{s a^{\prime}(s)}{a(s)} \stackrel{(1.7)}{=} \gamma\left(2 \mathfrak{n}_{f}\right)-1 \text {. }
$$

Therefore

$$
\frac{m_{2 \mathfrak{n}_{f}}^{k /\left(2 \mathfrak{n}_{f}\right)}}{m_{k}}<e^{\left(2 \mathfrak{n}_{f}-k\right)\left(\gamma\left(2 \mathfrak{n}_{f}\right)-1\right)}
$$

and

$$
\begin{aligned}
\frac{m_{2 \mathfrak{n}_{f}}}{m_{k}}|I|^{2 \mathfrak{n}_{f}-k} & <\exp \left\{-\left(2 \mathfrak{n}_{f}-k\right)\left(3+\gamma\left(2 \mathfrak{n}_{f}\right)-\gamma\left(2 \mathfrak{n}_{f}\right)+1\right)\right\} \\
& =\exp \left\{-4\left(2 \mathfrak{n}_{f}-k\right)\right\} \leq \exp \left\{-4 \mathfrak{n}_{f}\right\},
\end{aligned}
$$

proving the claim.

Proof of Claim 4.10. Let $c_{I}$ be the centre of the interval $I$, and let

$$
P_{2 \mathfrak{n}_{f}-1}(x)=\sum_{j=0}^{2 \mathfrak{n}_{f}-1} \frac{f^{(j)}\left(c_{I}\right)}{j !}\left(x-c_{I}\right)^{j}
$$

be the Taylor polynomial of $f$ at $c_{I}$. Then for $x \in I$

$$
f(x)=P_{2 \mathfrak{n}_{f}-1}(x)+\frac{f^{\left(2 \mathfrak{n}_{f}\right)}(\xi)}{\left(2 \mathfrak{n}_{f}\right) !}\left(x-c_{I}\right)^{2 \mathfrak{n}_{f}}, \quad \xi=\xi_{x} \in I,
$$

so that

$$
\left\|P_{2 \mathfrak{n}_{f}-1}\right\|_{I} \leq\|f\|_{I}+m_{2 \mathfrak{n}_{f}}\left(\frac{|I|}{2}\right)^{2 \mathfrak{n}_{f}} .
$$

For an arbitrary polynomial $S$ we have

$$
\left|S^{(k)}(0)\right| \leq(\operatorname{deg} S)^{k}\|S\|_{[-1,1]} .
$$

This is a relatively simple special case of V. Markov's inequalities see e.g. [14, Chapter VI, Sections 4.II and 4.III]). Using this inequality, we get

$$
\begin{aligned}
\left|f^{(k)}\left(c_{I}\right)\right| & =\left|P_{2 \mathfrak{n}_{f}-1}^{(k)}\left(c_{I}\right)\right| \\
& \leq\left(\frac{2}{|I|}\right)^{k}\left(2 \mathfrak{n}_{f}-1\right)^{k}\left\|P_{2 \mathfrak{n}_{f}-1}\right\|_{I} \\
& \leq\left(\frac{2}{|I|}\right)^{k}\left(2 \mathfrak{n}_{f}-1\right)^{k}\left[\|f\|_{I}+m_{2 \mathfrak{n}_{f}}\left(\frac{|I|}{2}\right)^{2 \mathfrak{n}_{f}}\right]
\end{aligned}
$$


and

$$
\begin{aligned}
\frac{\left|f^{(k)}\left(c_{I}\right)\right|}{e^{k} M_{k}} & \leq \frac{1}{m_{k}}\left(\frac{2}{|I|}\right)^{k}\left(\frac{2 \mathfrak{n}_{f}-1}{k}\right)^{k}\left[\|f\|_{I}+m_{2 \mathfrak{n}_{f}}\left(\frac{|I|}{2}\right)^{2 \mathfrak{n}_{f}}\right] \\
& \leq \frac{1}{m_{k}}\left(\frac{2}{|I|}\right)^{k} \exp \left(\frac{2 \mathfrak{n}_{f}-1}{e}\right)\left[\|f\|_{I}+m_{2 \mathfrak{n}_{f}}\left(\frac{|I|}{2}\right)^{2 \mathfrak{n}_{f}}\right] .
\end{aligned}
$$

Then using Corollary 2.3 from Bang's fundamental lemma and the definition of the Bang degree $\mathfrak{n}_{f}$, we obtain that

$$
B_{f}\left(c_{I}\right) \geq \min _{[0,1]} B_{f}>e^{-\mathfrak{n}_{f}-1} .
$$

Hence, for at least one $k, 0 \leq k \leq \mathfrak{n}_{f}$,

$$
\begin{aligned}
& e^{-\mathfrak{n}_{f}-1}<\frac{\left|f^{(k)}\left(c_{I}\right)\right|}{e^{k} M_{k}} \\
& \quad \leq \frac{1}{m_{k}}\left(\frac{2}{|I|}\right)^{k} \exp \left(\frac{2 \mathfrak{n}_{f}-1}{e}\right)\left[\|f\|_{I}+m_{2 \mathfrak{n}_{f}}\left(\frac{|I|}{2}\right)^{2 \mathfrak{n}_{f}}\right],
\end{aligned}
$$

whence

$$
m_{k}\left(\frac{|I|}{2}\right)^{k} \leq e^{\left(2+\frac{1}{e}\right) \mathfrak{n}_{f}}\left[\|f\|_{I}+m_{2 \mathfrak{n}_{f}}\left(\frac{|I|}{2}\right)^{2 \mathfrak{n}_{f}}\right]
$$

proving the claim.

It remains to spread estimate (4.7) from short to arbitrary sub-intervals $I \subset[0,1]$. We shall prove a bit more: we show that if the interval $I \subset[0,1]$ is not short, then

$$
\left(\frac{\Gamma\left(2 \mathfrak{n}_{f}\right)|I|}{|E|}\right)^{2 \mathfrak{n}_{f}}\|f\|_{E} \geq 1
$$

for any measurable subset $E \subset I$. Since $\|f\|_{I} \leq\|f\|_{[0,1]} \leq 1$, this does the job.

We fix a measurable subset $E \subset I$ where $I$ is not short, and choose a short sub-interval $I_{1} \subset I$ such that

$$
\left|E \cap I_{1}\right| \geq|E| \frac{\left|I_{1}\right|}{|I|},
$$

and

$$
m_{2 \mathfrak{n}_{f}}\left|I_{1}\right|^{2 \mathfrak{n}_{f}} \geq 2^{-2 \mathfrak{n}_{f}} e^{-2 \mathfrak{n}_{f}\left(3+\gamma\left(2 \mathfrak{n}_{f}\right)\right)}
$$


Existence of such $I_{1}$ follows by a straightforward dyadic argument. Then

$$
\begin{aligned}
& 1 \stackrel{(4.15)}{\leq}\left(2 e^{3+\gamma\left(2 \mathfrak{n}_{f}\right)}\right)^{2 \mathfrak{n}_{f}} m_{2 \mathfrak{n}_{f}}\left|I_{1}\right|^{2 \mathfrak{n}_{f}} \\
& \quad \stackrel{(4.5)}{\leq} \frac{1}{2}\left(2 e^{3+\gamma\left(2 \mathfrak{n}_{f}\right)}\right)^{2 \mathfrak{n}_{f}}\|f\|_{I_{1}} \\
& \stackrel{(4.7)}{\leq} \frac{1}{2}\left(2 e^{3+\gamma\left(2 \mathfrak{n}_{f}\right)}\right)^{2 \mathfrak{n}_{f}} \cdot 2\left(\frac{2 e\left|I_{1}\right|}{\left|E \cap I_{1}\right|}\right)^{2 \mathfrak{n}_{f}}\|f\|_{E \cap I_{1}} \\
& \stackrel{(4.14)}{<}\left(4 e^{4+\gamma\left(2 \mathfrak{n}_{f}\right)} \frac{|I|}{|E|}\right)^{2 \mathfrak{n}_{f}}\|f\|_{E}
\end{aligned}
$$

proving (4.13) and completing the proof of Theorem B.

\section{Variations on Bang's theme}

\subsection{Bang's differential inequality}

One can rewrite the fundamental inequality (2.2) as the differential inequality for the function

$$
L_{f}(x)=\log \frac{1}{B_{f}(x)} .
$$

If $B_{f}(x)$ is positive, then taking the logarithms in (2.2) and choosing there $q=\left[L_{f}(x)\right]+1$, we obtain

$$
L_{f}(x+h)>L_{f}(x)-e|h| A\left(L_{f}(x)+1\right) .
$$

Interchanging $x$ and $x+h$, we arrive at

Corollary 5.1.1. Suppose $f \in \mathscr{C}_{A}([0,1])$. Then the function $L_{f}:[0,1]$ $\rightarrow[0, \infty]$ is continuous and

$$
\left|L_{f}(x+h)-L_{f}(x)\right|<e|h| A\left(L_{f}(x)+1\right)
$$

whenever $x, x+h \in[0,1]$ and the values $L_{f}(x), L_{f}(x+h)$ are finite. In particular, if $L_{f}^{\prime}(x)$ exists, then

$$
\left|L_{f}^{\prime}(x)\right|<e A\left(L_{f}(x)+1\right) .
$$

It is remarkable that the function $L_{f}$ satisfies a simple differential inequality. Integrating this inequality, we get a reformulation of Corollary 2.3:

Corollary 5.1.3. Suppose $f \in \mathscr{C}_{A}([0,1])$. Then

$$
\int_{L_{*}+1}^{L^{*}+1} \frac{d s}{A(s)}<e
$$


where

$$
L_{*}=\min _{x \in[0,1]} L_{f}(x) \quad \text { and } \quad L^{*}=\max _{x \in[0,1]} L_{f}(x) .
$$

\subsection{One-sided version of the Denjoy-Carleman theorem}

Theorem 5.2.1. Suppose $f \in C^{\infty}([0,1])$ and

$$
\min _{[0,1]} f^{(j)} \geq-M_{j}, \quad j \in \mathbf{Z}_{+},
$$

where the sequence $\left\{M_{j}\right\}$ satisfies the quasianalyticity condition (1.5). If all derivatives of $f$ are non-negative at the origin, then they are non-negative everywhere on $[0,1]$.

Recall that $C^{\infty}([0,1))$-functions with all derivatives positive everywhere on $[0,1)$ are called absolutely monotonic. By the classical Bernstein theorem, every absolutely monotonic function on $[0,1)$ has an analytic extension to the unit complex disc.

Under a somewhat stronger assumption $f \in \mathscr{C}_{A}([0,1])$, this result was conjectured by Borel (see [6, p. 74]) and proved by Täcklind [22] and Bang [2].

For the proof of Theorem 5.2.1, we set

$$
B_{f}^{-}(x)=\max _{j \geq 0} \frac{\max \left\{-f^{(j)}(x), 0\right\}}{e^{j} M_{j}} .
$$

Repeating verbatim the proof of Lemma 2.1, we obtain that

$$
B_{f}^{-}(x+h)<\max \left\{B_{f}^{-}(x), e^{-q}\right\} e^{e h A(q)}
$$

for every $q \in \mathrm{N}$, every $x, x+h \in[0,1], h>0$. Then Theorem 5.2.1 readily follows from this estimate.

\subsection{Non-extendable quasianalytic functions}

If $f$ is a real analytic function on a closed interval $J$ (that is $f \in \mathscr{C}_{\left\{K^{j} j !\right\}}(J)$ with some constant $K$ ), then $f$ always has a real analytic extension on a larger interval $J^{\prime} \supset J$. In contrast, the Täcklind-Bang theorem combined with the Bernstein theorem give us examples of quasianalytically smooth functions defined on a closed interval which do not have a quasianalytically smooth extension; i.e. a smooth extension which belongs to a (probably, different) Denjoy-Carleman quasianalytic class on a larger interval.

We fix a logarithmically convex sequence $\left\{M_{j}\right\}$ satisfying (1.5) and such that

$$
\lim _{j \rightarrow \infty}\left(\frac{M_{j}}{j !}\right)^{1 / j}=+\infty
$$


and choose a positive sequence $\left\{c_{j}\right\}$ such that

$$
\lim _{j \rightarrow \infty} c_{j}^{1 / j}=1
$$

and

$$
\sum_{j=1}^{\infty} j^{n} c_{j} \leq M_{n}
$$

for all $n \in \mathrm{Z}_{+}$. For example, if $M_{n}=n !(\log n)^{n}$, then one can take

$$
c_{j}=\exp \left[-\frac{C j}{\log (j+e)}\right]
$$

with a proper choice of a positive constant $C$.

Then consider an even function

$$
f(x)=\sum_{k=0}^{\infty} c_{2 k} x^{2 k}
$$

which is is analytic in $(-1,1)$ and belongs to the quasianalytic class $\mathscr{C}_{\left\{M_{j}\right\}}$ on the segment $[-1,1]$. This function has no quasianalytically smooth extension on a larger interval. Otherwise, the extension would be an even function (by the Denjoy-Carleman theorem), and by the theorems of Täcklind-Bang and Bernstein it would have an analytic extension to a disk of radius larger than one. Clearly, this is impossible since the radius of convergence of the Taylor series which represents $f$ equals one.

This construction answers the question raised by P. Milman ${ }^{1}$.

\subsection{Bang's original version of the fundamental inequality}

Mention that Bang proved his results without assumption of the logarithmic convexity of the sequence $\left\{M_{j}\right\}$. He assumed that $M_{j}$ is the upper bound for the $\left|f^{(j)}(x)\right|$ on the closed interval $J$ and the sequence $\left\{M_{j}\right\}$ increases so rapidly that $M_{j}^{1 / j} \rightarrow \infty$. Then there exists a unique largest logarithmically convex

\footnotetext{
${ }^{1}$ A. Borichev indicated another construction of a non-extendable quasianalytic function. He considers the absolutely convergent series

$$
f(z)=\sum_{n} \frac{\delta_{n}}{z-\lambda_{n}}, \quad \lambda_{n}=1+\epsilon_{n}-i \delta_{n},
$$

with

$$
\epsilon_{n} \downarrow 0, \quad \epsilon_{n+1} / \epsilon_{n} \downarrow 0, \quad \delta_{n} \downarrow 0, \quad \delta_{n+1} / \delta_{n} \downarrow 0, \quad \delta_{n} / \epsilon_{n} \downarrow 0
$$

and using some results from [3] shows that under a special choice of these sequences the function has no quasianalytic extension to any larger interval $[-1-\gamma, 1+\gamma]$.
} 
minorant $M_{j}^{c} \leq M_{j}$. The equation $M_{j}^{c}=M_{j}$ is satisfied for infinitely many integers $j$, in particular for $j=0$ (see e.g. [14, Chapter 1]). This set of integers is denoted by P. Then Bang defines the 'norm'

$$
B_{f}(x)=\inf _{p \in \mathrm{P}} \max \left\{e^{-p}, \max _{0 \leq j \leq p} \frac{\left|f^{(j)}(x)\right|}{e^{j} M_{j}^{c}}\right\}
$$

and proves that if $B_{f}(x) \geq e^{-q}, q \in \mathrm{N}$, then

$$
B_{f}(x+h)<B_{f}(x) e^{e|h| A^{c}(q)}
$$

where

$$
A^{c}(q)=\frac{M_{q}^{c}}{M_{q-1}^{c}} .
$$

From here he deduces a more general version of Corollary 2.3 which already contains the result of Borel and Carleman formulated in the Introduction.

\subsection{Propagation of smallness for quasianalytically smooth functions}

Here, we give a simple 'global corollary' to Theorem B excluding the degree $\mathfrak{n}_{f}$ from estimate (1.8). We assume that $\mathscr{C}_{A}([0,1])$ is a regular quasianalytic Denjoy-Carleman class of functions, that is $A:[1, \infty) \rightarrow(0, \infty)$ is a nondecreasing $C^{1}$-function such that the integral $\int^{\infty} A^{-1}(s) d s$ is divergent and

$$
\gamma=\sup _{s \geq 1} \frac{s A^{\prime}(s)}{A(s)}<\infty
$$

We set

$$
\Omega(t) \stackrel{\text { def }}{=} \exp \left[-\frac{1}{e} \int_{1}^{\log (e / t)} \frac{d s}{A(s)}\right], \quad 0 \leq t \leq 1 .
$$

The function $\Omega$ steadily increases on the interval $[0,1], \Omega(0)=0$, and $\Omega(1)=$ 1.

A relative smallness of the set $E \subset[0,1]$ will be measured in the logarithmic scale by the quantity

$$
\alpha(E)=\frac{1}{3} \log ^{-1}\left(\frac{\Gamma}{|E|}\right)
$$

where as above $\Gamma=4 e^{4+\gamma}$.

Corollary 5.5.1. Suppose $\mathscr{C}_{A}([0,1])$ is a regular Denjoy-Carleman quasianalytic class, and suppose that $f \in \mathscr{C}_{A}([0,1])$. Then

$$
\Omega\left(\|f\|_{[0,1]}\right) \leq e \Omega\left(\|f\|_{E}^{\alpha(E)}\right) .
$$


In the real analytic case when $A(s)=C s, C$ is a positive constant, we have

$$
\Omega(t)=\exp \left[-\frac{1}{e C} \log \log \frac{e}{t}\right]=\left(\log \frac{e}{t}\right)^{-1 /(e C)} .
$$

Suppose that $\|f\|_{E} \leq \epsilon$. Then estimate (5.5.2) gives us

$$
\|f\|_{[0,1]} \leq e \epsilon^{\alpha(E) e^{-e C}} .
$$

Certainly, estimate (5.5.3) can be obtained by classical means using a complex extension with control over the uniform norm and the two-constants-theorem [13], [23], or by an elementary real variable technique [17].

However, already in the logarithmic Denjoy-Carleman class when $A(s)=$ Cs $\log (s+e)$, the Corollary gives a new result.

Proof of Corollary 5.5.1. We have

$$
\|f\|_{[0,1]} \leq\left(\frac{\Gamma}{|E|}\right)^{2 \mathfrak{n}_{f}}\|f\|_{E}
$$

or

$$
\begin{aligned}
1 \leq \exp \left\{2 \mathfrak{n}_{f} \log \left(\frac{\Gamma}{|E|}\right)+\log \frac{1}{\|f\|_{[0,1]}}\right\}\|f\|_{E} \\
<\exp \left\{3 \mathfrak{n}_{f} \log \left(\frac{\Gamma}{|E|}\right)\right\}\|f\|_{E}=\exp \left\{\frac{\mathfrak{n}_{f}}{\alpha(E)}\right\}\|f\|_{E},
\end{aligned}
$$

that is

$$
e^{-\mathfrak{n}_{f}} \leq\|f\|_{E}^{\alpha(E)} .
$$

Since

$$
\begin{aligned}
\log \Omega\left(\|f\|_{[0,1]}\right)-\log \Omega\left(e^{-\mathfrak{n}_{f}}\right) & =\frac{1}{e} \int_{\log \|f\|_{[0,1]}^{-1}+1}^{\mathfrak{n}_{f}+1} \frac{d s}{A(s)} \\
& \leq \frac{1}{e} \sum_{\log \|f\|_{[0,1]}^{-1}<j \leq \mathfrak{n}_{f}} \frac{M_{j-1}}{M_{j}}<1,
\end{aligned}
$$

we finally get

$$
\Omega\left(\|f\|_{[0,1]}\right)<e \Omega\left(e^{-\mathfrak{n}_{f}}\right)<e \Omega\left(\|f\|_{E}^{\alpha(E)}\right),
$$

completing the proof. 


\section{REFERENCES}

1. Bang, Th., Om quasi-analytiske funktioner, Kjøbenhavn, Nyt Nordisk Forlag, 1946.

2. Bang, Th., The theory of metric spaces applied to infinitely differentiable functions, Math. Scand. 1 (1953), 137-152.

3. Beurling, A., Quasianalyticity. Mittag-Leffler Lectures on Complex Analysis (1977-1978), Collected Works, Vol I, Birkhäuser, Boston, 1989.

4. Brudnyi, Yu., and Ganzburg, M., An extremal problem for polynomials of $n$ variables, Izv. AN SSSR (Mat) 37 (1973), 344-355 (Russian).

5. Brudnyi, A., Local inequalities for plurisubharmonic functions, Ann. of Math. 149 (1999), 511-533.

6. Carleman, T., Fonctions quasi analytiques, Paris, Gauthier Villars, 1926.

7. Cohen, P., A simple proof of the Denjoy-Carleman theorem, Amer. Math. Monthly 75 (1968), 26-31.

8. Dudley, R. M., and Randol, B., Implications of pointwise bounds on polynomials, Duke Math. J. 29 (1962), 455-458.

9. Ilyashenko, Yu., and Yakovenko, S., Counting real zeros of analytic functions satisfying linear ordinary differential equations, J. Differential Equations 126 (1996), 87-105.

10. Ilyashenko, Yu., Hilbert type numbers for Abel equations, growth and zeros of holomorhpic functions, Nonlinearity 13 (2000), 1337-1342.

11. Hirschman, I. I., On the distribution of zeros of functions belonging to certain quasianalytic classes, Amer. J. Math. 72 (1950), 396-406.

12. Hörmander, L., The Analysis of Linear Partial Differential Operators, I, Berlin, Springer Verlag, 1983.

13. Lavrentiev, M. M., Some improperly posed problems of mathematical physics, Berlin, Springer Verl., 1967.

14. Mandelbrojt, Sz., Séries adhérentes, régularisation des suites, applications, Gauthier-Villars, Paris, 1952.

15. Matsaev, V., and Sodin, M., Asymptotics of the Fourier and Laplace transforms in weighted spaces of analytic functions, Algebra \& Analysis 14 no. 4 (2002), 100-134. English transl. in St. Petersburg Math. J. 14 no. 4 (2003).

16. Nadirashvili, N., On a generalization of Hadamard's three-circle theorem, Vestnik Mosk. Un-ta. (Mat.) 31 no. 3 (1976), 39-42.

17. Nadirashvili, N., Estimating solutions of elliptic equations with analytic coefficients bounded on some sets, Vestnik Mosk. Un-ta. (Mat.) 34 no. 2 (1979), 42-46.

18. Novikov, D., and Yakovenko, S., A complex analog of the Rolle theorem and polynomial envelopes of irreducible differential equations in the complex domain, J. London Math. Soc. (2) 56 (1997), 305-319.

19. Remez, E. J., Sur une propriéte des polynômes de Tschebycheff, Commun. Inst. Sci. Kharkov 13 (1936), 93-95.

20. Roytvarf, N., Bernstein type inequalities and finiteness properties of analytic functions, Ph.D. Thesis, Weizmann Inst. of Sciences, Rehovot, 1996.

21. Roytvarf, N., and Yomdin, Y., Bernstein classes, Ann. Inst. Fourier 47 (1997), 825-858.

22. Täcklind, S., Sur les classes quasianalytiques des solutions des équations aux dérivées partielles du type parabolique, Thèse, Uppsala, 1936.

23. Vessella, S., A continuous dependence result in the analytic continuation problem, Forum Math. 11 (1999), 695-703. 
24. Yomdin, Y., Global Finiteness Properties of Analytic Families and Algebra of their Taylor Coefficients, Fields Inst. Commun. 24 (1999), 527-555.

DEPARTMENT OF MATHEMATICS

MICHIGAN STATE UNIVERSITY

EAST LANSING, MI 48824

USA

E-mail: fedja@math.msu.edu

DEPARTMENT OF MATHEMATICS

MICHIGAN STATE UNIVERSITY

EAST LANSING, MI 48824

USA

E-mail: volberg@math.msu.edu
SCHOOL OF MATHEMATICAL SCIENCES

TEL AVIV UNIVERSITY

RAMAT AVIV, 69978

ISRAEL

E-mail: sodin@post.tau.ac.il 